\title{
Synthesis and properties of novel materials at high pressure and temperature-molecular dynamics simulation studies
}

\author{
S L CHAPLOT \\ Condensed Matter Physics Division, Bhabha Atomic Research Centre, Mumbai 400 085, India.
}

\begin{abstract}
Molecular dynamics simulations, in combination with lattice dynamics studies, based on semiempirical interatomic potentials, have been very useful in the study of properties of complex novel materials at high temperature and pressure. Various properties such as the equation of state, elastic and thermodynamic properties, phase transitions and melting have been studied. These studies help in understanding the synthesis of important new and novel materials, especially the amorphous materials, compounds with unusually coordinated atoms, (e.g. with five-coordinated silicon atoms), materials with controlled thermal expansion, etc. A few examples will be discussed from our recent studies.
\end{abstract}

Keywords. Molecular dynamics simulation; lattice dynamics studies; novel materials.

\section{Introduction}

Molecular dynamics (MD) simulation and lattice dynamics are well established techniques used in the atomic level study of the structure and dynamics, phase transitions and thermodynamic properties of solids (Rao and Chaplot 1979; Chaplot 1986, 1994, 1997; Allen and Tildesley 1987; Rao et al 1987). Simulations complement experiments; they help unravel microscopic phenomena that are often only indirectly revealed from experiments (see e.g. Chaplot and Sikka 1993a,b; Chaplot et al 1998). Also, experiments are needed to validate the simulations. Simulations of thermal or high pressure processes are useful to study the synthesis of novel materials, crystal growth etc.

In this review we discuss a few examples of our studies involving simulations and lattice dynamics of certain important materials. Section 2 deals with some aspects of the techniques. In $\S 3$, we focus on the synthesis of amorphous materials through application of both static and shock pressures. We have carried out the shock simulations very recently (Chaplot and Sikka 1998). Lattice dynamics also has an important role in understanding the crystal to amorphous phase transition and the free energies of associated crystalline phases. The simulations bring out the properties of the structure, heating effects, irreversibility of the phase transitions etc.

Next, in $\S 4$, we describe our studies on the high pressure-temperature phase transitions of major mantle minerals, the magnesium silicate in the enstatite and perovskite phases and, perhaps the first simulation of seismic discontinuties at mantle conditions of pressures and temperatures (Chaplot and Choudhury 1998). Most interestingly, the MD simulations reveal the synthesis of a novel five-coordinated silicate phase which might characterize the transition zone between the Earth's upper and lower mantles, at a depth of $440-660 \mathrm{~km}$. Synthesis of a five-coordiated calcium silicate phase has been reported (Angel et al 1996) from recent experiments which may be compared with our above predictions on magnesium silicate. The simulations also reveal the role of defects in phase transitions and melting (Chaplot et al 1998), especially in perovskite in the lower mantle and help understand the striking stability of the orthorhombic perovskite over the huge lower mantle, from the depth of $660-2700 \mathrm{kms}$.

Section 5 deals with the almost unique property of a novel material, $\mathrm{ZrW}_{2} \mathrm{O}_{8}$, which shows a large isotropic negative thermal expansion (NTE) over an exceptionally wide temperature range of 0-1050 K (Mary et al 1996). The NTE is a consequence of the quantum nature of phonons alongwith some unusual properties of the material. Through detailed lattice dynamics calculations (Mittal and Chaplot 1998), we are able to understand the NTE in $\mathrm{ZrW}_{2} \mathrm{O}_{8}$, at a quantitative level, in terms of the role of transverse acoustic modes and certain low energy librational and translational phonons. The calculations show an elastic instability at a fairly low pressure of $0.6 \mathrm{GPa}$ which suggests a possible mechanism of the reported phase transition in $\mathrm{ZrW}_{2} \mathrm{O}_{8}$ from a cubic to an orthorhombic phase under low pressure. The orthorhombic phase shows an NTE only below $300 \mathrm{~K}$ (Hu et al 1998) which is also understood in the framework of the lattice dynamical calculations.

A summary is presented in $\S 6$. It appears that simulations based on a suitable tailored semiempirical 
model may provide unique microscopic understanding of the thermodynamic properties and synthesis processes.

\section{Complementary role of lattice dynamics and molecular dynamics simulations}

As is well known, the dynamics of atoms in a solid at low temperature involve small amplitude vibrations about the equilibrium atomic positions. These vibrations described in terms of a set of independent harmonic oscillators, called phonons, of frequencies $\omega_{i}$, where $i$ runs over all the phonon branches and the wavevectors in the Brillouin zone. The quantum nature of atomic vibrations is most apparent in the low temperature specific heat which deviates significantly from its classical value of 3R below the Debye temperature. Perhaps even more dramatic consequence of the quantum nature of dynamics is on the thermal expansion which sometimes has anamolous behaviour as will be discussed in $\S 5$. Standard techniques of lattice dynamics are used to calculate the phonon properties including the low temperature specific heat and thermal expansion. The pressure as a variable may be treated in the quasiharmonic approximation. The low temperatures in the context of lattice dynamics imply temperatures below the Debye temperature which is typically above $700 \mathrm{~K}$ for many ceramic oxides, silicates, minerals etc.

At high temperatures, the atomic dynamics can be described classically. The technique of molecular dynamics (MD) simulation is very useful which involves solution of the classical equations of motion of the nuclear coordinates. The forces on atoms may be evaluated either by first principles electronic energies calculations, or by using empirical interatomic potentials. The MD method is very convenient in examining the microscopic structure, anharmonic dynamics, diffusion, mechanisms of phase transitions, effects of lattice defects etc. The simulation is also very useful at low temperatures although one should note that the consequences of the quantum nature of the dynamics are inhibited while treating the nuclear coordinates via classical equations of motion.

Having made these remarks, we now discuss below a few interesting examples of our lattice dynamics and $\mathrm{MD}$ studies. While our studies on quartz and $\mathrm{AlPO}_{4}$ have been based on semiempirical potentials as available in the literature (Tsuneyuki et al 1989; Van Beest et al 1990), for several more complex materials we have derived empirical interatomic potentials which have been tested on a range of observed structural and dynamical properties (see e.g. Chaplot 1997; Chaplot et al 1998; Choudhury et al 1998).

\section{Static pressure and shock induced amorphization}

Amorphous or glassy materials are usually formed by rapid quenching of a melt. However, in case of a large number of compounds, especially those composed of polyhedral networks such as quartz, application of pressure often leads to a crystal to amorphous transition (Sikka and Gupta 1998). Typically, static or shock pressures of about 2-20 GPa are involved. Molecular dynamics simulations are useful in understanding, and even predicting, such transformations (Tsuneyuki et al 1989; Chaplot and Sikka 1990, 1991, 1993a,b; Tse and Klug 1991). Soft phonon instabilities, as calculated from lattice dynamics, have also been associated with the mechanisms of such transformations (Chaplot and Sikka 1990, 1991, 1993a,b).

Earlier we carried out MD simulations under static pressures on quartz, $\mathrm{AlPO}_{4}$ and $\mathrm{LiKSO}_{4}$, which clearly showed the first-order crystal to amorphous transitions (Chaplot and Sikka 1990, 1991, 1993a,b). While in the parent crystalline phases the atoms $\mathrm{Si}, \mathrm{Al}$ and $\mathrm{Li}$ are known to have a four-fold tetrahedral coordination of oxygen atoms, the MD simulations revealed that in the transformed amorphous phase there is a mixture of 4 , 5 and 6-fold coordinated atoms at high pressures (figure 1). The average coordination number further increases with increasing pressure and reduces with decrease of pressure. The average coordination number in quartz glass reduced from about 5.3 to about 4.5 on release of pressure which is close but different from the quartz glass prepared from melt. However, in case of $\mathrm{AlPO}_{4}$ the amorphization transition is found to be reversible with a hysteresis, in agreement with reported experiments. The MD simulations also showed that the transition pressure decreased with increase of temperature. As in case of usual MD simulations, the changes in the structural variables with pressure including the equation of state are also obtained. These simulated values in the parent crystalline phase revealed that the bond angles and the bond lengths reach certain limiting values before the event of transformation begins.

Lattice dynamics calculations as a function of pressure in both quartz and $\mathrm{AlPO}_{4}$ showed that, prior to the amorphization pressure, a zone boundary phonon mode at the K-point becomes soft (figure 2) (Chaplot and Sikka 1990, 1991, 1993a,b). Molecular dynamics simulations, corresponding to the soft phonon mode, revealed the presence of a transient high pressure phase involving a super structure of the ambient pressure phase (Chaplot and Sikka 1991, 1993a,b). The transient phase thus predicted from lattice dynamics and MD simulations was expected to occur as an intermediate crystalline phase between the ambient pressure crystalline phase and the pressure-induced amorphous phase. It is gratifying that such an intermediate crystalline phase was indeed observed after our prediction and showed superlattice peaks characteristic of a superstructure (Kingma et al 1993). Recently, Wentzcovitch et al (1998) have determined the structure of the intermediate phase from 
first principles calculations and confirmed that the new structure derives from our predicted soft phonon instability and quantitatively explains the experimental data of Kingma et al (1993). The first principles calculations also provide support for the semiempirical pair potential which was used in the lattice dynamical calculations.
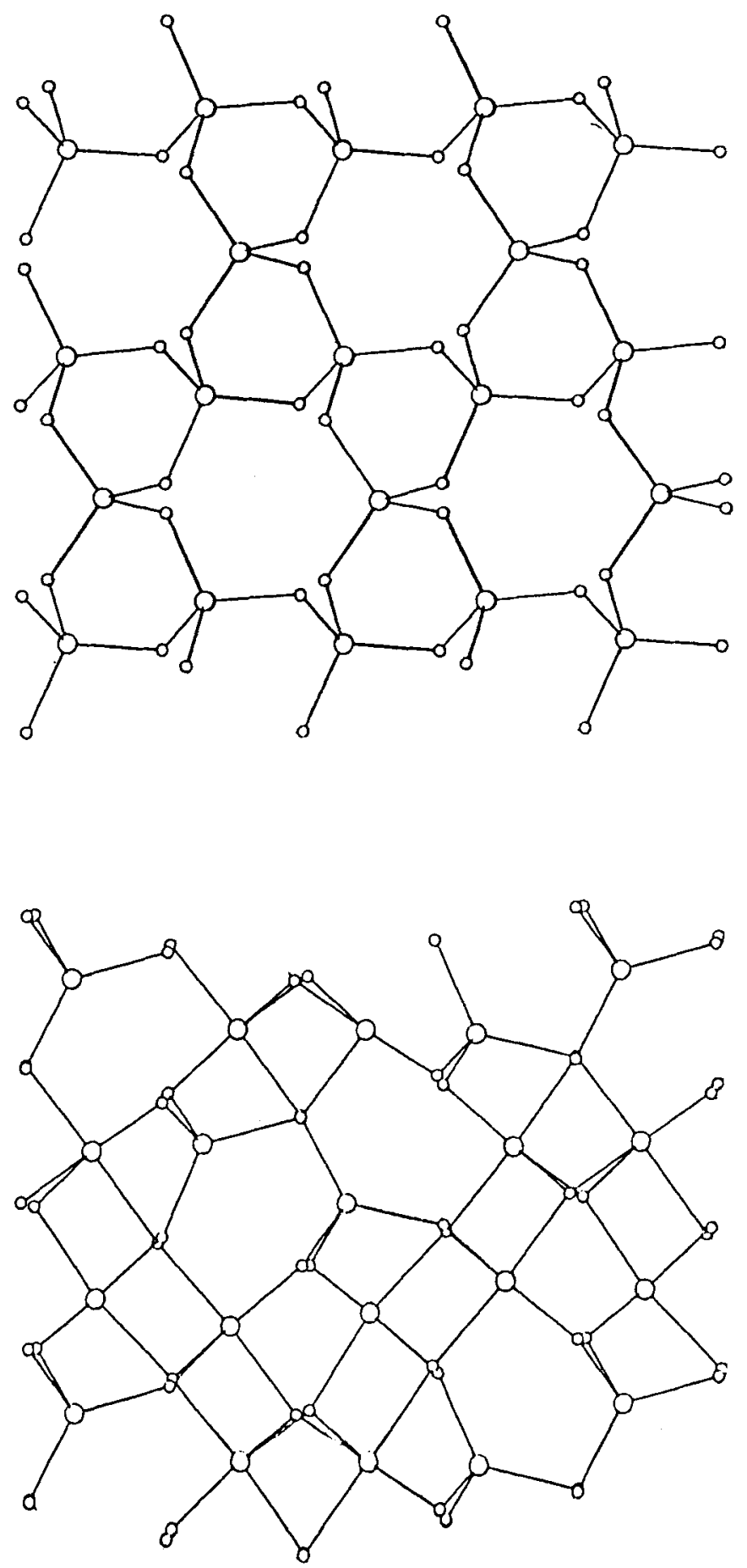

Figure 1. A snap shot of the atomic arrangement at $20 \mathrm{GPa}$ (above) in alpha-quartz, and (below) after pressure-induced amorphization. The bigger and smaller circles denote the silicon and oxygen atoms respectively (Chaplot and Sikka 1993a)
Recently, we have carried out a MD simulation of shock pressure induced amorphization of quartz. A very long periodic macrocell of $0.2 \mu \mathrm{m}$ length is used along the shock direction which contains about 13000 atoms. These large scale MD simulations have been performed on BARC-parallel computer ANUPAM. Figure 3 shows the shock pressure front, temperature and the average coordination of the silicon atoms along the shock-axis after $10 \mathrm{ps}$ of the shock initiation in one of the simulations. These simulations reveal a very interesting result, namely, that the shock-induced transformations may occur in a short time scale of a few ps, which is, however, two orders greater than the shortest vibrational period of about $30 \mathrm{fs}$. This time scale is much shorter than the time scale of a ns often deduced from typical shock experiments, which may be limited by the shortest time scale of observation in those experiments. The kinetics in shock-induced amorphization is also found to be much faster than that found in static pressure-induced amorphization (Chaplot and Sikka 1993a). This is partly due to an important difference in shock experiments compared to the static pressure experiments, namely, that the temperature of the sample increases significantly in the former. Moreover, the nature of the shock pressure, which is nonhydrostatic in the initial stage near the shock front, greatly facilitates the amorphization transformation.

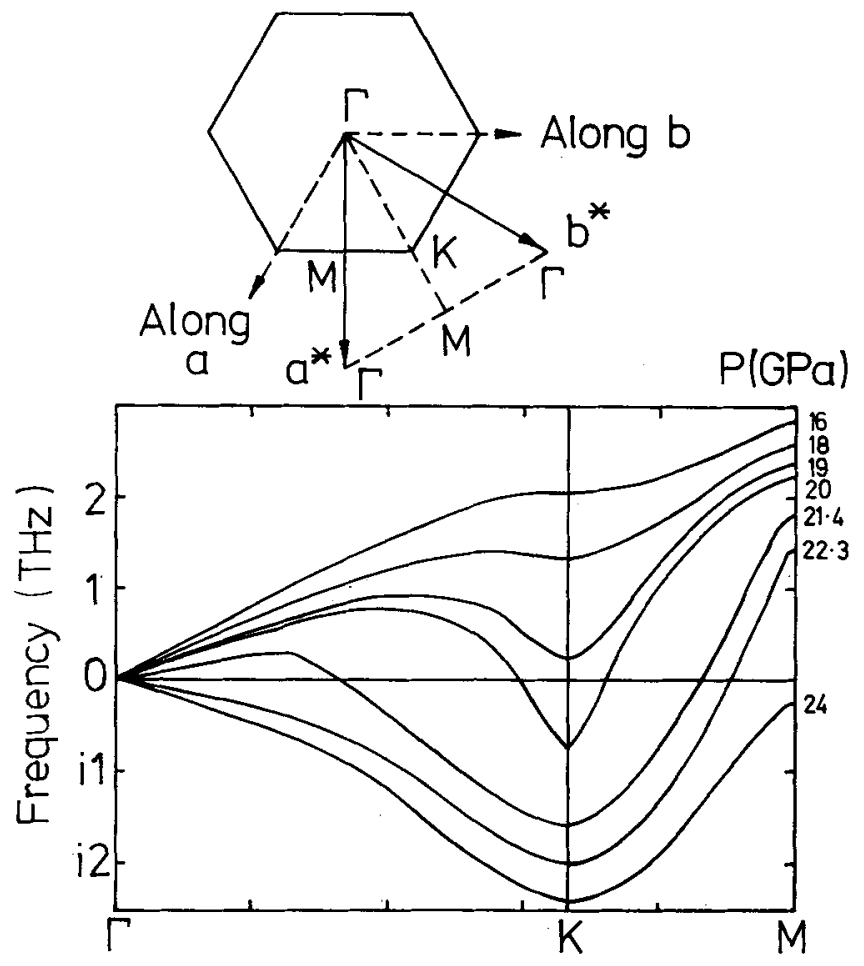

Figure 2. The soft phonon branch in the alpha-quartz structure as a function of pressure. A section of the Brillouin zone is also shown (Chaplot and Sikka 1993b). 
Consistent with our previous MD simulation as a function of static pressure and temperature, we find that the shock induced amorphization occurs at a lower pressure (at about $14 \mathrm{GPa}$ ) compared to the static pressure-induced amorphization (at about $20 \mathrm{GPa}$ ) due to a high temperature of about $1500 \mathrm{~K}$ under shock. The results are in very good agreement with expectations, which gives a lot of confidence in our procedure of shock simulation. Such shock simulations using realistic Coulomb and short-ranged interatomic potentials have perhaps been reported for the first time. Details of this work will be published elsewhere.

\section{Prediction of a five-coordinated silicate phase and seismic discontinuities in Earth's mantle}

Magnesium silicate in its various polymorphic forms constitutes a major component of the Earth's mantle which extends down to a depth of $2700 \mathrm{~km}$ (Dziewonski and Anderson 1981; Jeanloz and Thompson 1983). The upper mantle, upto a depth of $440 \mathrm{~km}$, contains the olivin, pyroxene, spinel and garnet phases, while the lower mantle from $660 \mathrm{~km}$ downwards is believed to be essentially made of the orthorhombic perovskite phase. One of the most important differences in the upper and lower mantle silicate crystal structures is in the coordination of the silicon atoms which are respectively 4 and 6 . The magnesium coordination is also increasing from 6 to 8 but somewhat less sharply since there is a large variation in $\mathrm{Mg}-\mathrm{O}$ bond lengths from 2.0 to $2.4 \AA$, unlike the $\mathrm{Si}-\mathrm{O}$ bond lengths which are rather well defined around 1.63 and $1.80 \AA$ for the 4- and 6-fold coordinations, respectively. Information about the transition zone from $440 \mathrm{~km}$ to $660 \mathrm{~km}$ is rather limited; it probably contains mixtures of 4- and 6-fold Si-coordinated phases. This kind of information is only indirectly derived from seismic velocity data and laboratory experiments on relationships of the structures and their properties. An accurate modelling of mantle minerals is therefore of utmost importance to understanding Earth's interior while it is also a major challenge in condensed matter physics.

Figure 4 shows the seismic discontinuities in the longitudinal and transverse acoustic wave velocities and density across the mantle boundaries. We have carried out MD simulation as a function of pressure and temperature starting with the ortho-enstatite $\mathrm{Mg}_{2} \mathrm{Si}_{2} \mathrm{O}_{6}$ as the ambient phase which is one of the important constituents in the upper mantle and involves 4-fold coordination of $\mathrm{Si}$ atoms. In simulations at a fixed temperature of $900 \mathrm{~K}$, enstatite transformed to a new phase involving 5-coordinated silicon at $13 \mathrm{GPa}$, which then further transformed at $17 \mathrm{GPa}$ to the orthorhombic perovskite phase involving 6-coordinated $\mathrm{Si}$ atoms. Both the transitions involve density discontinuities of about
$10 \%$ (figure 4 ), which are very close to the observed discontinuities at the mantle boundaries. Figure 4 also shows our calculation of the acoustic velocities from large scale MD simulations of fairly long wave length (about $40 \AA$ ) acoustic phonons, which also shows a very encouraging and satisfactory agreement with the mantle data.
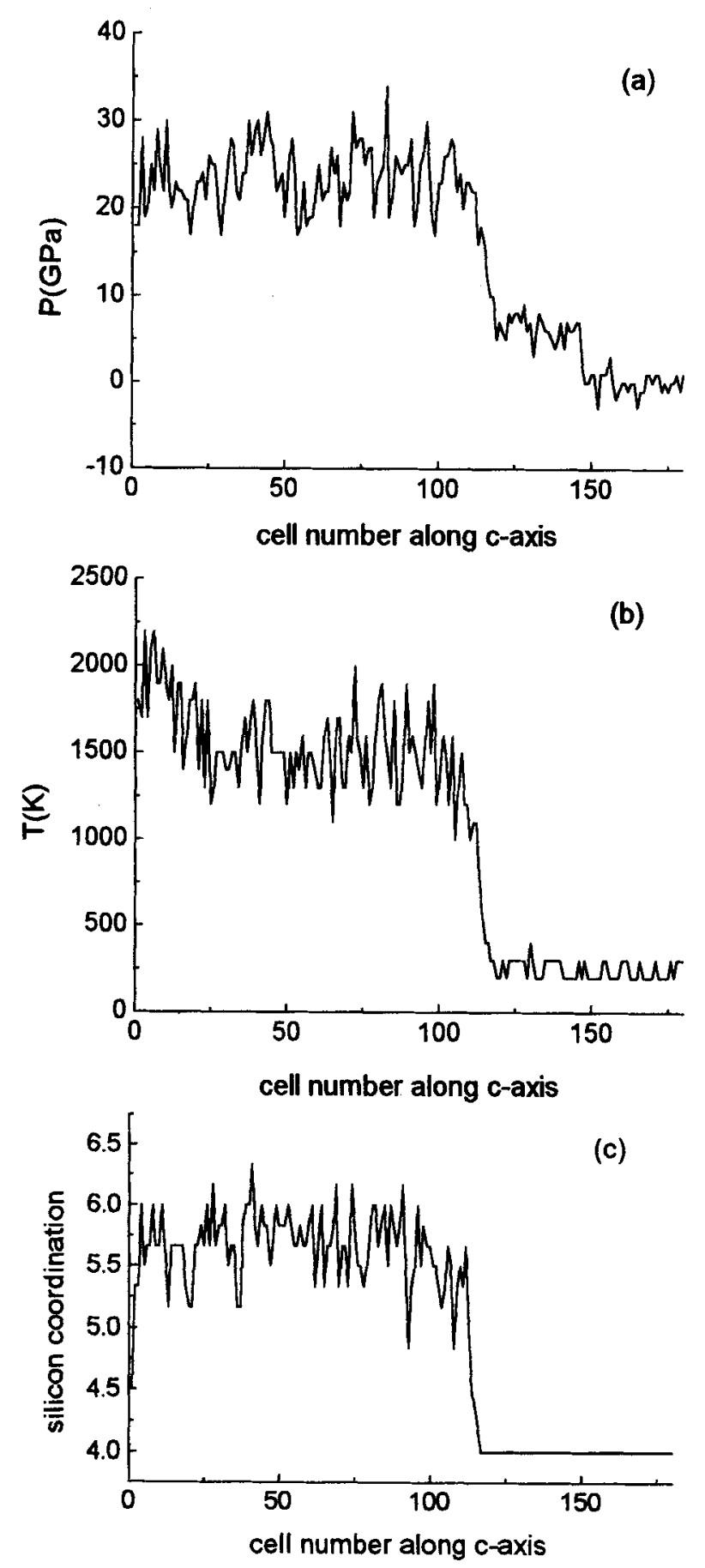

Figure 3. An MD simulation of shock-induced amorphization of alpha-quartz. Various physical parameters are shown along the shock-direction after $10 \mathrm{ps}$ of the start of the shock. 


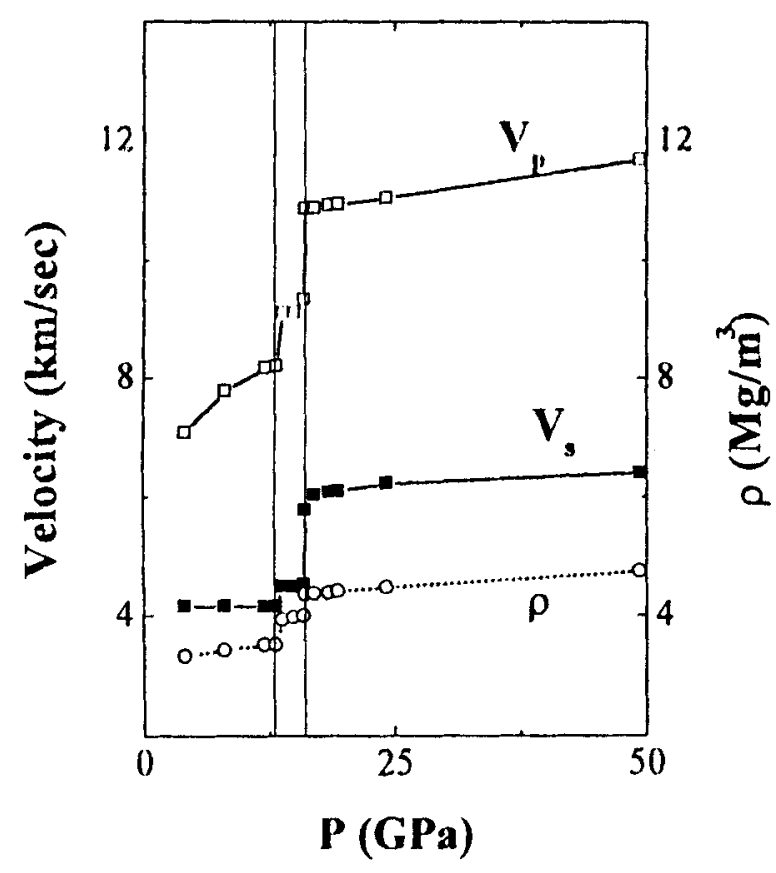

\section{P (GPa)}

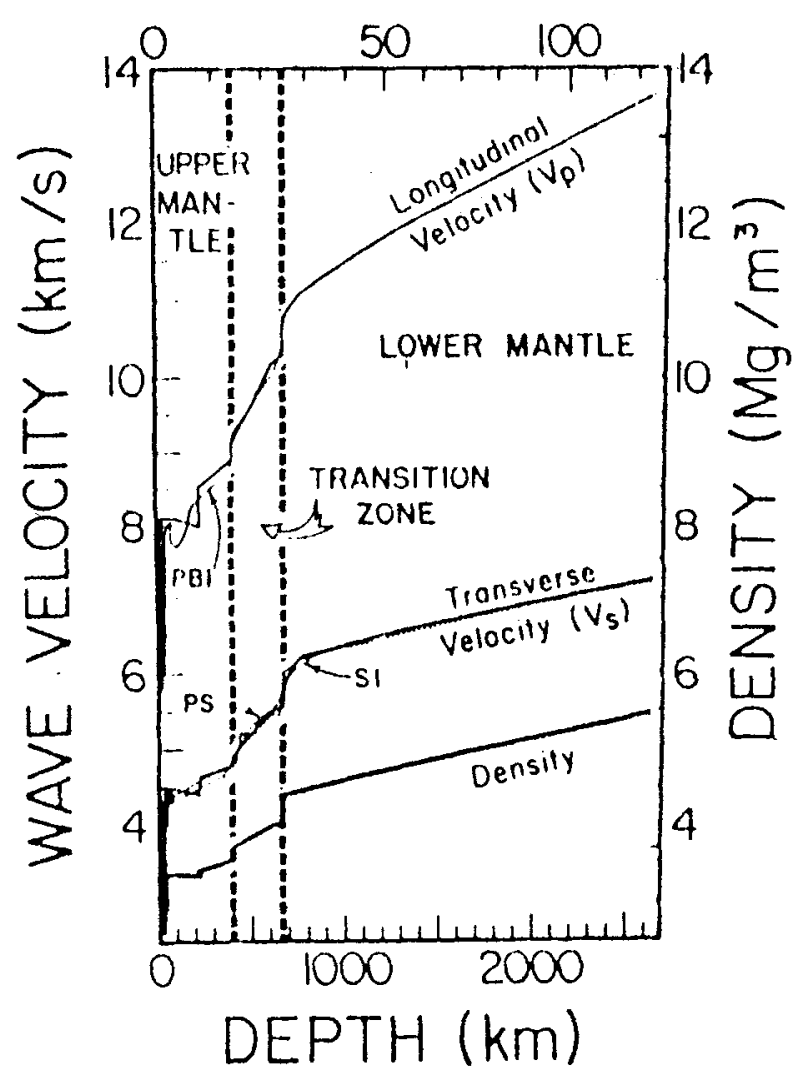

Figure 4. Seismic discontinuities in Earth's mantle; (below) observed (Dziewonski and Anderson 1981, Jeanloz and Thompson 1983); (above) calculated (Chaplot and Choudhury 1998). Note the difference in the pressure scale in the two figures.
Considering a rather complex mantle composition, our simulations appear to be somewhat simplistic involving only one initial phase. However, these are perhaps the first simulations of the seismic velocities and the phase transitions from an upper mantle phase to a lower mantle phase at realistic high pressures and temperatures. Already these simulations, based on empirical interatomic potentials, have used several weeks of CPU time on a parallel supercomputer; therefore, first principles simulations would be presently too time consuming. We hope that MD simulations would also be performed on other initial phases such as garnets and olivine. However, as seen from the present simulations, enstatite appears to be a good representative of the upper mantle phases in terms of the microscopic atomic structure and local coordinations, and therefore these simulations are able to provide a reasonable description of the seismic discontinuities. The simulations also suggest that the predicted 5coordinated silicate phase may be a good representative of the transition zone. As mentioned above when the $\mathrm{Si}$ coordination is 4 or 6 , the $\mathrm{Si}-\mathrm{O}$ bond lengths are very close to 1.6 or $1.8 \AA$, respectively. However, in the 5 -coordinated silicate, the $\mathrm{Si}-\mathrm{O}$ bonds are distributed between 1.6 and $1.9 \AA$. The same feature is also observed in a laboratory synthesized $\mathrm{CaSi}_{2} \mathrm{O}_{5}$ phase which has some of the silicon atoms as 5-coordinated (Angel et al 1996). The present simulations suggest that 5-coordinate silicon phases may be synthesized by high pressures and high temperatures.

\section{Large isotropic negative thermal expansion in $\mathrm{ZrW}_{2} \mathrm{O}_{8}$ over $\mathbf{0 - 1 0 5 0 ~ K}$}

While negative thermal expansion (NTE) is known to occur in several materials, in complex structures and ceramics it usually occurs in only certain directions, and over limited range of temperatures. Therefore, the observation of a large isotropic NTE in cubic $\mathrm{ZrW}_{2} \mathrm{O}_{8}$ over its full stability range upto $1050 \mathrm{~K}$ (Mary et al 1996) offers interesting possibilities of using this material in composites with desired values of thermal expansion. As thermal expansion is closely associated with anharmonic phonons, we have tried to understand the NTE by lattice dynamical calculations. As explained earlier, the phonomena is essentially quantum mechanical in nature and therefore could not be addressed adequately by MD simulations involving classical trajectories of atoms.

The contribution of each phonon of frequency $\omega_{i}$ to the volume thermal expansion is equal to

$$
\frac{1}{B V} \Gamma_{\mathrm{i}} C_{\mathrm{v}}\left(\omega_{\mathrm{i}}, T\right) \text {, }
$$

where $\Gamma_{\mathrm{i}}$ is the mode Gruneisen parameter $\left(=-\partial \ln \omega_{\mathrm{i}} /\right.$ $\partial \ln V), C_{\mathrm{v}}\left(\omega_{\mathrm{i}}, T\right)$ is the contribution of this mode to 
the specific heat, $B$ the bulk modulus, $V$ volume of the unit cell, and $i$ runs over all the phonon branches and the wave vectors in the Brillouin zone. Figure 5 gives our calculation of NTE in cubic $\mathrm{ZrW}_{2} \mathrm{O}_{8}$ which shows a very good agreement with the published experimental data. The deviation in the cubic phase above $400 \mathrm{~K}$ is due to a volume drop associated with a disordering transition not accounted in the calculation. The same figure also includes a comparison of the calculated and observed thermal expansion in a high pressure orthorhombic phase which shows NTE only below $300 \mathrm{~K}$. The excellent description of the thermal expansion in both the cubic and orthorhombic phases also strongly validates our lattice dynamical model for such thermodynamical calculations. The model is based on empirical Coulomb and short range interatomic potentials derived from the static and dynamic equilibrium conditions of the crystal structure.

A detailed analysis of the individual phonon contribution leads to a very interesting result that, in the cubic phase, about $40 \%$ of the NTE at $300 \mathrm{~K}$ arose from the two transverse acoustic branches and most of the remaining contribution came from the low energy phonons below $8 \mathrm{meV}$, which constitute only $6 \%$ of the total phonon modes in the crystals. These phonons essentially involve translational and librational modes of the $\mathrm{ZrO}_{6}$ and $\mathrm{WO}_{4}$ units which remain rigid during these low energy vibrations. The high energy phonons, especially above $60 \mathrm{meV}$, contribute to lowering the magnitude of the NTE at high temperatures above $200 \mathrm{~K}$. In the orthorhombic phase, the contribution of the low energy phonons is much reduced such that the net value of the thermal expansion becomes positive above $300 \mathrm{~K}$.

As stated above, the TA phonons are especially highly anharmonic and contribute significantly to the NTE. The

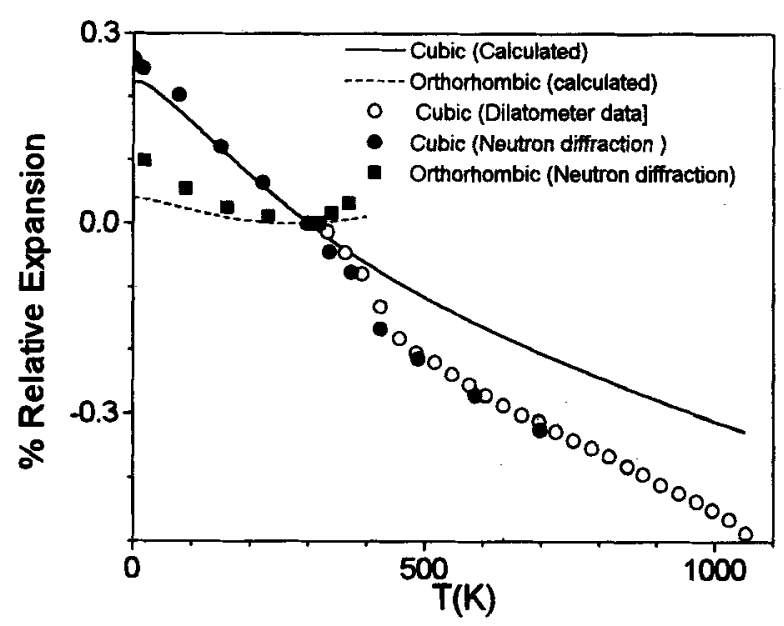

Figure 5. The comparison of the observed thermal expansion in the cubic (Mary et al 1996) and the orthorhombic (Hu et al 1998) phases of $\mathrm{ZrW}_{2} \mathrm{O}_{8}$ with lattice dynamical calculations (Mittal and Chaplot 1998). same anharmonicity manifests in their softening at $0.6 \mathrm{GPa}$ (figure 6) leading to an elastic instability of the cubic phase. This result suggests that the elastic instability of the cubic phase is a likely driving force in the cubic to orthorhombic transition observed ( $\mathrm{Hu}$ et al 1998) at $0.3 \mathrm{GPa}$.

One may state that the NTE arises since the frequencies of a number of phonons reduce very significantly on compression of the crystal, quite unlike in normal materials. This is very well illustrated in our detailed lattice dynamical calculations.

\section{Summary and outlook}

In this review we have discussed examples of our recent computational studies involving lattice dynamics and $\mathrm{MD}$ simulations of complex structure materials at high pressures and temperatures. We have introduced, perhaps a new technique, of simulating the shock-induced phase transitions, which is important because a lot of very high pressure experiments have been possible with shock pressures when high static pressures were more difficult to apply. Even at low pressures, for example, in case of pressure-induced amorphous silica, the equation of state has been derived only from shock experiments. A lot of further work is needed on shock simulations. There is a possibility of studying synthesis of new materials through application of shock pressures.

The first simulations of phase transitions across Earth's upper to lower mantle, and the associated seismic discontinuities, appear to be very satisfying and of fundamental significance to Earth modelling. Their importance lies in the fact that much of our knowledge about Earth's interior is based on seismic data and their modelling based on limited laboratory experiments. The simulations have also brought out the possibility of synthesizing a

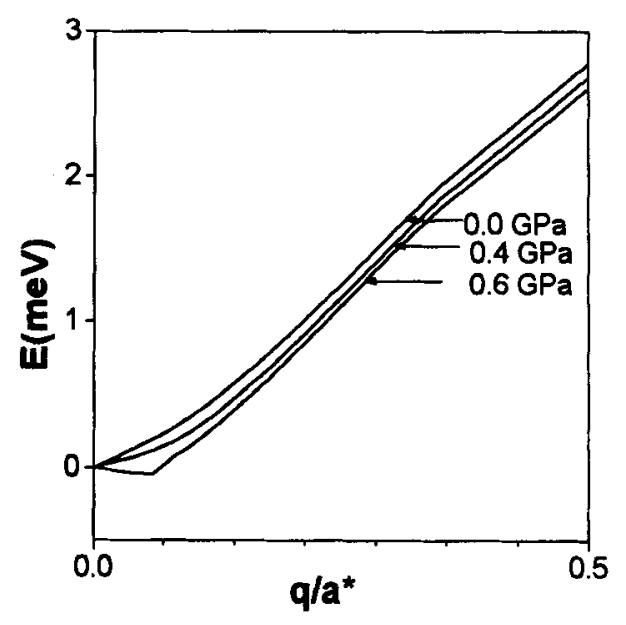

Figure 6. The softening of a transverse acoustic branch as a function of pressure in cubic $\mathrm{ZrW}_{2} \mathrm{O}_{8}$ indicating an elastic instability (Mittal and Chaplot 1998). 
novel five-coordinated silicate phase which may have properties characteristic of Earth's transition zone.

Our detailed calculation of the negative thermal expansion in the ambient and high pressure phase of $\mathrm{ZrW}_{2} \mathrm{O}_{8}$ brings out the important unique role of lattice dynamics. Lattice dynamical studies have also been known to be important in predicting phase transitions and understanding the driving force and mechanism of phase transitions besides other material properties. The number of lattice dynamics studies seems to be on a decline during the last decade or so perhaps due to difficulties in modelling complex structures; this trend needs to be reversed.

\section{References}

Allen M P and Tildesley D J 1987 Computer simulation of liquids (Oxford: Clarendon)

Angel R J, Ross N L, Seifert F and Fliervoet T F 1996 Nature 384441

Chaplot S L 1986 Curr. Sci. 55949

Chaplot S L 1994 Indian J. Pure \& Appl. Phys. 32560

Chaplot S L 1997 Bull. Mater. Sc. 20739

Chaplot S L and Sikka S K 1990 Solid State Phys (India) C33 328

Chaplot S L and Sikka S K 1991 Proc. XIII AIRAPT int. conf. high pressure science and technology, Bangalore (ed.) A K Singh (New Delhi: Oxford and IBH publishing) p. 259

Chaplot S L and Sikka S K 1993a Phys. Rev. B47 5710

Chaplot S L and Sikka S K 1993b Phys. Rev. Lett. 712674

Chaplot S L and Sikka S K 1998 unpublished
Chaplot S L and Choudhury N 1998 Solid State Phys. (India) C41

Chaplot S L, Choudhury N and Rao K R 1998 Am. Mineral. 83937

Choudhury N, Ghose S, Chowdhury C P, Loong C K and Chaplot S L 1998 Phys. Rev. 58756

Dziewonski A M and Anderson D L 1981 Phys. Earth Planet. Int. 25297

Hu Z, Jorgensen J D, Teslic S, Short S, Argyriou D N, Evans J S O and Sleight A W 1998 Physica B241-243 370

Jeanloz R and Thompson A B 1983 Rev. Geophys. Space Phys. 2151

Kingma K K, Hemley R J, Mao H and Veblen D R 1993 Phys. Rev. Lett. 703927

Mary T A, Evans J S O, Vogt T and Sleight A W 1996 Nature 90272

Mittal R and Chaplot S L 1998 Solid State Phys. (India) C41

Rao K R and Chaplot S L 1979 in Current trends in lattice dynamics (ed.) K R Rao (Bombay: Indian Physics Association) p. 589

Rao K R, Chaplot S L, Choudhury N, Ghose S and Price D L 1987 Science 23664

Sikka S K and Gupta S C 1998 in Shock compression of condensed matter-1997 AIP Conf. Proc. (eds) S C Schmidt, D D Dandekar and J W Forbes (New York: American Institute of Physics) 429 p. 145

Tsuneyuki S, Matsui Y, Aoki H and Tsukada M 1989 Nature 339209

Tse J S and Klug D D 1991 Phys. Rev. Lett. 673559

van Beest B W H, Kramer G J and van Santen R A 1990 Phys. Rev. Lett. 641955

Wentzcovitch R M, de Silva C, Chelikowsky J R and Binggeli N 1998 Phys. Rev. Lett. 802149 